\title{
Bus Data Acquisition and Remote Monitoring System Using Gsm \& Can
}

\author{
Narisetty Mallikarjuna Rao ${ }^{1}, \mathrm{H}$. Raghunatha Rao ${ }^{2}$ \\ ${ }^{I} P G$ Student (M. Tech), Dept. of ECE, Chirala Engineering College, Chirala , A.P, India \\ ${ }^{2}$ Associate Professor, Dept. of ECE, Chirala Engineering College, Chirala, A.P, India.
}

\begin{abstract}
Automotive industry uses CAN as the in-vehicle network (IVN) for the engine management, the body electronics like door and roof control, air conditioning, and lightning, as well as for the entertainment control. The American and the Far East passenger car manufacturers have also started implementing CAN-based car automation. CAN networks used in engine management connect several electronic control units (ECUs). Most of the passengers cars are equipped with CAN-based multiplex systems connecting body electronic ECUs. These multiplex networks link door and roof control units as well as lighting control units and seat control units. The different CAN-based IVNS are connected via gateways1. In many system designs, the gateway functionality is implemented in the dashboard. The dashboard itself may equipped with a local CAN network connecting the different displays and instruments.
\end{abstract}

Keywords: GSM, CAN, Adhoc Network; data acquisition; transmission network, Temperature monitoring.

\section{Introduction}

Many data acquisition systems are prerequisites to realizing equipment control, status monitoring and fault diagnosis. The traditional signal acquisition systems are mostly by wire, i.e., the sensor connected acquisition system by cable, when measuring point is dispersed and hidden, especially when we control and monitor large complex equipment, it needs a large number of cumbersome cabling work, and each measurement can only be concentrated in one area, otherwise, it has a very long connection cable, and a long connection cable will introduce a large number of noise and interference [3-7].For the traditional wiring methods not only affect the reliable operation of equipment, but also extend signal acquisition time, so it is difficult to meet safety requirements. However, the signal acquisition system based on wireless transmission will be able to overcome these shortcomings. The signal acquisition system based on wireless transmission is that the signals which acquired from the sensor have $\mathrm{A} / \mathrm{D}$ conversion and then transmitted to $\mathrm{PC}$ for processing through wireless transmission. So we designed a signal acquisition system based on wireless transmission. Next, the system's structure and principle, hardware and software are introduced.

This paper presents the design proposal of hardware and software of information terminal (a machine) and wireless receiver module of multi-point short-range wireless data collection and transmission network as shown in fig.1, which provides a low-powered and high-performance wireless data communication system.

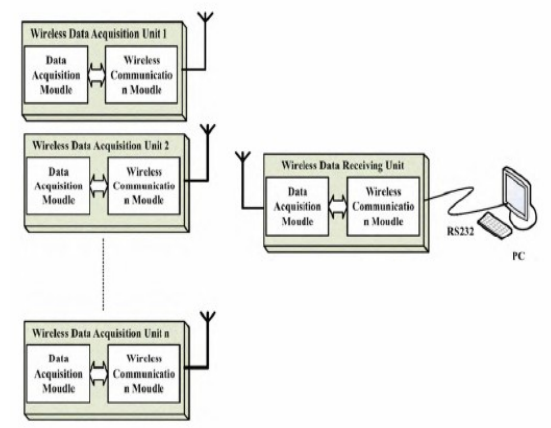

Figure 1 An Example of Data Acquisition System

The intention behind the design of short-range wireless communication network is to provide short-distance broadband wireless access to mobile environment or formulation of temporary network; it is the further development of internet in mobile environment. The main advantage of short-range wireless communication network is lower cost and more flexible use.

The signal acquisition system based on wireless transmission consists of a number of wireless data acquisition units and a wireless data receiver unit, which can realize single path and multi-path signal acquisition. The system's overall structure is shown in Fig 1. 
The wireless data acquisition unit includes data acquisition module and wireless transmission module. It connects an external signal acquisition sensor. This unit acquires signals according to user's given sampling frequency and points, and then transmits signal data to the receiver unit by wireless way.

In actual operation, the wireless data acquisition unit fixes near measured area through magnet or other fixed ways, thus it can reduce the length of connecting cable between sensors and the acquisition system, reduces the difficulty of cabling work, and increases the reliability of signal. All of the data transmitted by the nodes will be collect by the central server and is displayed in a user friendly format. The Software is much intelligent and generates signals on occurrence of fault to make the user attention. The wireless data receiver unit mainly consists of a microcontroller and a wireless transmission module, which connects to the receiver through zigbee, and communicates with wireless data acquisition units through wireless transmission. It receives the data from other modules through CAN bus and transmits to receiver. The receiver then sends the status as SMS through GSM modem.

\section{Implementation of the Design}

The wireless data acquisition unit includes several things like wireless transmission module and data acquisition module. The data acquisition module consists of sensor power supply circuit, a micro controller, A/D conversion chip, extended SRAM, watchdog circuit and power circuit.

Using wireless transmission module can realize Wireless communication. In order to shorten the development cycle, we adopt single-chip radio frequency integrated circuit and a single ARM Controller. Zigbee is a specification for a suite of high level communication protocols using small, low-power digital radios based on the IEEE 802.15.4,2006 standard for wireless personal area networks (WPANs),such as wireless headphones connecting with cell phones via short-range radio

During the data acquisition module Circuit designing, taking into account the board size and power consumption, we try to use low power and small package device. So we use The LPC2148 consists of an ARM7TDMI-S CPU with emulation support, the ARM7 Local Bus for interface to on-chip memory controllers, the AMBA Advanced High-performance Bus (AHB) for interface to the interrupt controller, and the VLSI Peripheral Bus (VPB, a compatible superset of ARM's AMBA Advanced Peripheral Bus) for connection to onchip peripheral functions. The LPC2148 configures the ARM7TDMI-S processor in little-endian byte order. AHB peripherals are allocated a 2 megabyte range of addresses at the very top of the 4 gigabyte ARM memory space. Each AHB peripheral is allocated a 16kB address space within the AHB address space.

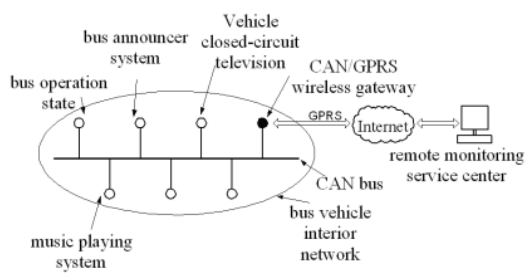

Figure 2 The Design Scheme

As Shown in Figure 2, bus vehicle interior network structure can use topology bus network, all the electrical systems nodes inside the bus vehicle are attached to the CAN bus through their own respective CAN communication module. CAN / GSM Wireless Gateway not only be used to organize and coordinate all the interior electrical systems nodes, but also can be used as internal and external communications data interface. It gathered up the information from all the interior electrical systems nodes sent to the remote monitoring service center via GSM wireless module.

Topology bus network structure can be used within bus vehicle interior network. The network use two cables as a transmission medium. All the electrical system nodes connected directly to the CAN bus through the corresponding hardware interface. All nodes share the same data channel. The message a node send out can be received multiple nodes on the CAN bus. The bus vehicle interior topology network is as shown in Figure 3. Taking CAN nodes' performance and cost into account, using ST's STM32F103RBT6 chip, its internal integrated CAN module, support CAN protocol 2.0A and 2.0B active mode, baud rate up to $1 \mathrm{Mbit} / \mathrm{sec}$. 


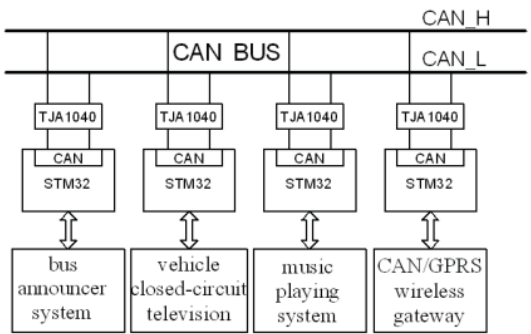

Figure 3 Interior Topology Network

It has been designed to manage high number of incoming messages efficiently with a minimum CPU load. It also meets the priority requirements for transmit messages. In today's CAN applications, the number of nodes in a network is increasing and often several networks are linked together via gateways. Typically the number of messages in the system (and thus to be handled by each node) has significantly increased. the diagram of CAN node hardware circuit is as shown in Figure 4.

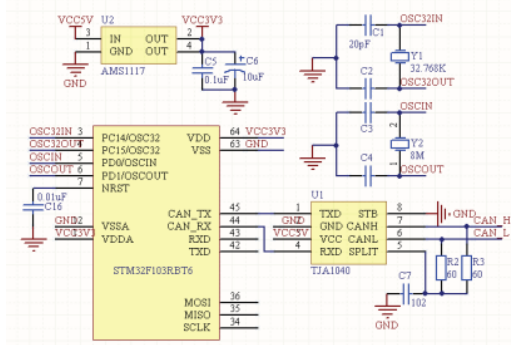

Figure 4 Hardware Circuit

The block diagram of the implemented system is shown in Fig. 5. CAN node can send and receive data on CAN bus, through drivers program, so that multimedia information systems can be used on bus as possible. CAN node can receive control commands from other nodes, as well as send useful information to the CAN bus.

Initialization: To enter Initialization mode the software sets the INRQ bit in the CAN_MCR register and waits until the hardware has confirmed the request by setting the INAK bit in the CAN_MSR register. To leave Initialization mode, the software clears the INQR bit. beCAN has left Initialization mode once the INAK bit has been cleared by hardware. However the Rx line has to be in recessive state to leave this mode. While in Initialization mode, all message transfers to and from the CAN bus are stopped and the status of the CAN bus output CANTX is recessive (high).Entering Initialization Mode does not change any of theconfiguration registers.To initialize the CAN Controller,software has to set up the Bit Timing registers and the filter banks. If a filter bank is not used, it is recommended to leave it non active (leave the corresponding FACT bit in the CAN_FCRx register cleared).

\section{Transmission handling:}

a) In order to transmit a message, the application must select one empty transmit mailbox, set up the identifier, the data length code (DLC) and the data before requesting the transmission by setting the corresponding TXRQ bit in the CAN_MCSR register. Once the mailbox has left empty state, the software no longer has write access to the mailbox registers. Immediately after the TXRQ bit has been set, the mailbox enters pending state and waits to become the highest priority mailbox, see Transmit Priority. As soon as the mailbox has the highest priority it will be scheduled for transmission. The transmission of the message of the scheduled mailbox will start (enter transmit state) when the CAN bus becomes idle. Once the mailbox has been successfully transmitted, it will become empty again. The hardware indicates a successful transmission by setting the RQCP and TXOK bits in the CAN_MCSR and CAN_TSR registers.

b) If the transmission fails, the cause is indicated by the ALST bit in the CAN_MCSR register in case of an Arbitration Lost, and/or the TERR bit, in case of transmission error detection.

c) A transmission request can be aborted by the user setting the ABRQ bit in the CAN_MCSR register. In pending or scheduled state, the mailbox is aborted immediately. An abort request while the mailbox is in transmit state can have two results. If the mailbox is transmitted successfully the mailbox becomes empty with the TXOK bit set in the CAN_MCSR and CAN_TSR registers. If the transmission fails, the mailbox becomes scheduled, the transmission is aborted and becomes empty with TXOK cleared. In all cases the mailbox will become empty again at least at the end of the current transmission. 


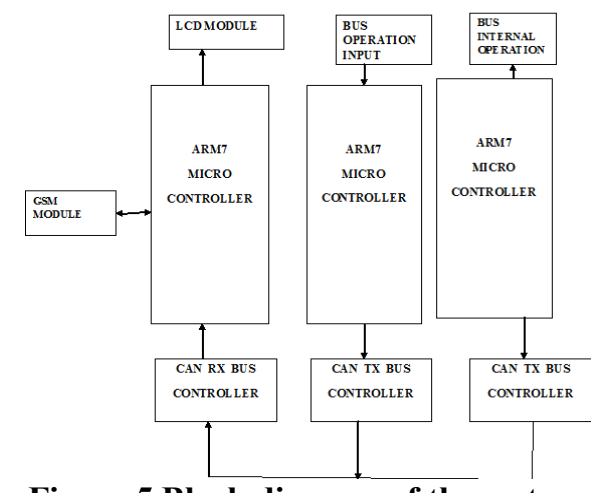

Figure 5 Block diagram of the system

The same Procedure is followed for reception of the signals. It is explained in the following lines.

a) For the reception of CAN messages, three mailboxes organized as a FIFO are provided. In order to save CPU load, simplify the software and guarantee data consistency, the FIFO is managed completely by hardware. The application accesses the messages stored in the FIFO through the FIFO output mailbox.

b) A received message is considered as valid when it has been received correctly according to the CAN protocol (no error until the last but one bit of the EOF field) and It passed through the identifier filtering successfully.

c) On the storage of the first message in the FIFO - FMP[1:0] bits change from $0 \mathrm{~b} 00$ to $0 \mathrm{~b} 01$-an interrupt is generated if the FMPIE bit in the CAN_IER register is set. When the FIFO becomes full (i.e. a third message is stored) the FULL bit in the CAN_RFR register is set and an interrupt is generated if the FFIE bit in the CAN_IER register is set. On overrun condition, the FOVR bit is set and an interrupt is generated if the FOVIE bit in the CAN_IER register is set.

\section{Results \& Conclusions}

In this paper the design and development of A low-powered and high-performance wireless data communication system was designed with the principle of CAN-GSM and ARM7 series of single-chip computer as the core hardware are demonstrated. An available solution to the wireless data communications was put forward, and this solution was good at stronger real-time response, higher reliability requirement and smaller data amount, which is widely applied various fields such as data communications, environmental monitoring and security Guard System.

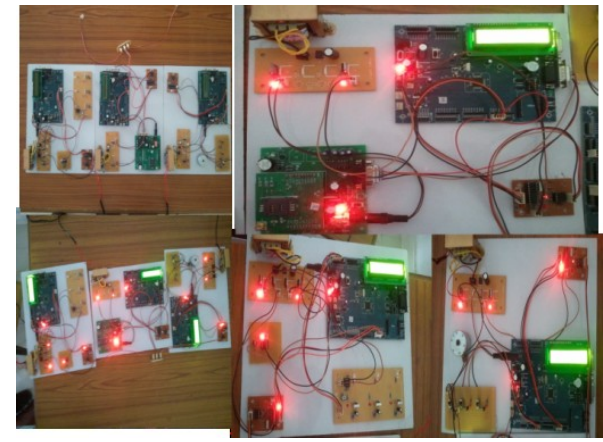

Figure 6 Designed Data acquisition system

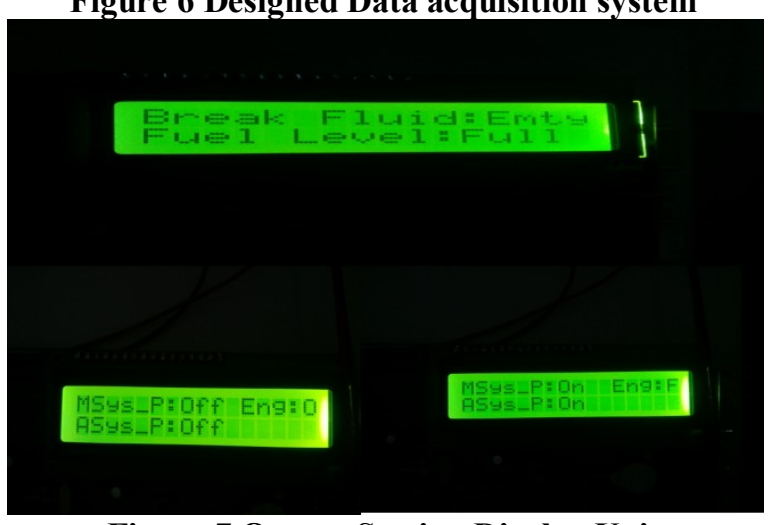

Figure 7 Output Section Display Unit 


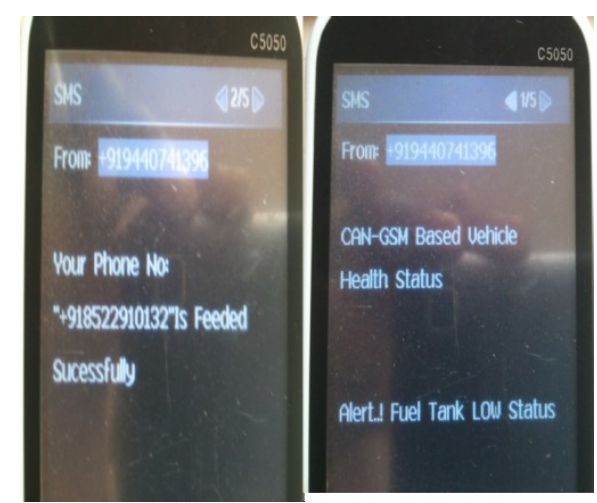

Figure 8 Output at the Mobile Base station

\section{Acknowledgements}

The authors would like to thank the anonymous reviewers for their comments which were very helpful in improving the quality and presentation of this paper.

\section{References:}

[1] A.Bonastre,J.V.Capella and J.herrero. A CAN fieldbus based architecture for dis-tributed control systems implementation, Proceedings of the 5th IFAC Int.Conference on Fieldbus Systems and their Applications, 2003.

[2] W.Lawrenz $\square$ From Theory to Practical lications $\square$ Springer Publishers CAN System Engineering $\square$ 1997 $\square$ 1-9

[3] Cena.Gianluca,Valenzano and Adriano. Improved CAN fieldbus for industrial applications, IEEE Transactions on Industrial Elcctronics, 1997.x(4):553-564.

[4] Etschberger K. CAN-based higher layer protocols andprofiles[A]. Proceedings of the 4-th International CANConference[C]. Berlin 1997. [5] An-Ping Wang;Hou-Sheng Hsu,Pau-Lo Hsu. Remote monitoring and control via the intelligent database gateway for the CAN-based wheelchair,Networking,Sensing and Control, 2004.3:445-450.

[5] A.Bonastre,J.V.Capella and J.herrero. A CAN fieldbus based architecture for dis-tributed control systems implementation, Proceedings of the 5th IFAC Int.Conference on Fieldbus Systems and their Applications, 2003.

[6] W.Lawrenz $\square$ From Theory to Practical lications $\square$ Springer Publishers CAN System Engineering $\square$ 1997 $\square$ 1-9

[7] Cena.Gianluca,Valenzano and Adriano. Improved CAN fieldbus for industrial applications, IEEE Transactions on Industrial Elcctronics, 1997.x(4):553-564.

[8] Etschberger K. CAN-based higher layer protocols andprofiles[A]. Proceedings of the 4-th International CANConference[C]. Berlin 1997. [5] An-Ping Wang;Hou-Sheng Hsu,Pau-Lo Hsu. Remote monitoring and control via the intelligent database gateway for the CAN-based wheelchair,Networking,Sensing and Control, 2004.3:445-450.

[9] http://batescomponents.com/catalog/parts/78LE54-2 4.html

[10] Popa, M.Popa, A.S., Cretu, V., Micea, M., "Monitoring Serial Communications in Microcontroller Based Embedded Systems Computer Engineering and Systems", The 2006 International Conference on, Nov. 2006 Page(s):56 - 61

[11] Computational Intelligence in Scheduling (SCIS 07), IEEE Press, Dec. 2007, pp. 57-64, doi:10.1109/SCIS.2007.357670

\section{Authors Profile:}

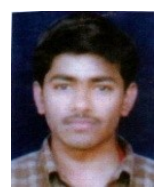

Narisetty Mallikarjuna Rao is a PG Student. He is pursuing his M.Tech degree from Dept. of ECE, Chirala Engineering College, Chirala with specialization in VLSI \& Embadded Systems.

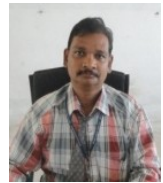

H. RAGHUNATHA RAO is working as an Associate Professor in the department of Electronics \& Communication Engineering in Chirala Engineering College, Chirala. completed masters from JNTUK. He has over 14years of teaching experience. 